\title{
O impacto da pandemia por COVID-19 na qualidade de vida de pacientes com doenças crônicas não transmissíveis
}

\author{
Ayllin Doria Werneck Caetano Lourenço ${ }^{a}$, Marcella Rezende Mattos Coutinho ${ }^{\mathrm{a}}$ \\ aCurso de Medicina do Centro Universitário Serra dos Órgãos (UNIFESO), Teresópolis, RJ, Brasil.
}

RESUMO A pandemia do novo Coronavírus despertou medo e incertezas, potencializando a vulnerabilidade de pessoas portadoras de Doenças Crônicas não Transmissíveis (DCNT), ao inseri-las em um grupo de maior risco para a forma grave da doença. Estudos apontam que as medidas de segurança epidemiológicas afetaram os portadores de DCNT, particularmente naqueles de baixa renda, contribuindo para o aumento da pobreza, redução da prática regular de atividades físicas e alteração dos hábitos alimentares. O cenário atual colocou em pauta a necessidade de políticas públicas voltadas para a qualidade de vida da população, tendo em vista que estas interferem diretamente no estado de saúde do indivíduo. Dante disto, objetivou-se com esta revisão discutir o impacto causado pela pandemia na qualidade de vida de pacientes portadores de DCNTs e apresentar novas perspectivas em relação ao cuidado com esses pacientes.

PALAVRAS-CHAVE COVID-19; doenças crônicas não transmissíveis; educação em saúde; pandemia; qualidade de vida

Aceito 21 de abril de 2021 Publicado online 30 de maio de 2021

Cite este artigo: Lourenço ADWC, Coutinho MRM (2021) O impacto da pandemia por COVID-19 na qualidade de vida de pacientes com doenças crônicas não transmissíveis. Multidisciplinary Reviews 4: e2021014. DOI: 10.29327/multi.2021014

\section{The impact of the COVID-19 pandemic on the quality of life of patients with chronic non-}

\section{transmissible diseases}

ABSTRACT The pandemic of the new Coronavirus raised fear and uncertainty, increasing the vulnerability of people with Chronic Non-Transmissible Diseases (NCDs) by placing them in a higher risk group for the severe form of the disease. Studies show that the epidemiological security measures have affected the carriers of NCDs, particularly in those of low income, contributing to increased poverty, reduced regular physical activity practice, and changes in eating habits. The current scenario has put on the agenda the need for public policies aimed at the quality of life of the population, considering that these directly interfere with the individual's health status.

KEYWORDS: chronic noncommunicable diseases; COVID-19; health education; pandemic; quality of life

\section{Introdução}

As Doenças Crônicas não Transmissíveis (DCNT) constituem um conjunto de condições crônicas, de ordem multifatorial, início gradual e prognóstico incerto. As DCNT representam a principal causa de mortalidade no Brasil e decorrem do efeito da "transição epidemiológica", no qual consiste em suas fases finais, da "cronificação das doenças", decorrente de um aumento da expectativa média de vida.

A Organização Mundial da Saúde (OMS) inclui como doenças crônicas: doenças do aparelho circulatório (cerebrovasculares, cardiovasculares), neoplasias, doenças respiratórias crônicas e diabetes mellitus (World Health Organization 2020).

As DCNT provocam diversos impactos, dentre eles o aumento dos gastos em saúde, tratamento prolongado, afastamento do trabalho, aumento do estado de pobreza das famílias, aumento da necessidade de internações, o que afeta diretamente a qualidade de vida dos pacientes. 
A qualidade de vida é um tema de grande importância, o qual precisa ser discutido e colocado entre as prioridades das políticas públicas, uma vez que sua avaliação permite elencar um planejamento de estratégias de prevenção.

De acordo com a OMS, "qualidade de vida" é "a percepção do indivíduo de sua inserção na vida, no contexto da cultura e sistemas de valores nos quais ele vive e em relação aos seus objetivos, expectativas, padrões e preocupações". Este conceito subjetivo engloba a saúde física, estado psicológico, relações pessoais e o nível de independência do indivíduo, que em geral, pode ser influenciada direta ou indiretamente por fatores relacionados à doença (Azevedo et al. 2013).

A fisiopatologia associada ao agravamento da infecção causada pelo SARS-CoV-2 aponta a participação da enzima conversora de angiotensina-2 (ECA2) nas células endoteliais presentes no coração, rins e nos pulmões. Sendo assim, a regulação positiva dessa enzima ocorre em pacientes com doenças cardiovasculares, diabetes e hipertensão que fazem tratamento com inibidores da ECA2 e bloqueadores dos receptores da angiotensina II do tipo I, contribuindo para o crescimento exponencial de células infectadas e a rápida evolução da COVID-19 nessa população, assim como em pacientes com DPOC e asma, uma vez que o sistema respiratório é o alvo do vírus (Estrela et al. 2020).

A pandemia causada pelo SARS-CoV-2 potencializou a vulnerabilidade das pessoas que vivem com doenças crônicas não transmissíveis, ao colocá-las como grupo de risco para agravamento da nova doença. A alta prevalência de pessoas com DCNTs levou ao aumento da letalidade dos casos de COVID-19, reiterando a necessidade de políticas públicas e sociais para proteger a população.

Como um dos principais fatores de risco para casos graves, a Divisão de Vigilância das Doenças e Agravos Não Transmissíveis (DIVDANT) recomendou que pacientes com DCNTs sejam estimulados à prática de atividade física e alimentação saudável como fatores de reforço positivo para imunidade (Teixeira 2020).

Objetivou-se com esta revisão discutir o impacto causado pela pandemia na qualidade de vida de pacientes portadores de DCNTs e apresentar novas perspectivas em relação ao cuidado com esses pacientes.

\section{Métodos}

Trata-se de uma revisão da literatura integrativa, na qual foi realizada uma busca nas bases indexadas de dados do MEDLINE/PubMed" (National Institutes of Health), SciELO (Scientific Electronic Libray Online), EBSCOhost e Google Acadêmico, utilizando os descritores na lingua inglesa: "Chronic noncommunicable diseases", "COVID-19, "quality of life", "pandemic", "health education". Foram incluídos artigos no idioma português e inglês, no período entre janeiro de 2013 a dezembro de 2020. Dezesseis artigos foram selecionados a partir da leitura dos mesmos e compatibilidade do conteúdo com o tema.

\section{Resultados e Discussão}

Segundo o Guia de Vigilância Epidemiológica de Síndromes Respiratórias Agudas COVID-19 publicado em 5 de agosto de 2020, as DCNTs tais como a obesidade, hipertensão arterial, cardiopatias e pneumopatias, doenças renais crônicas, neoplasias, anemia falciforme e talassemia, são consideradas fatores de risco para agravamento da COVID-19 (Ministério da Saúde 2020).

As DCNT são responsáveis por cerca de $70 \%$ de todas as mortes no mundo, estimando-se 38 milhões de mortes anuais. Estudos apontam que as DCNTs afetam em maior proporção as populações de baixa renda, por estarem mais expostas aos riscos e terem menor acesso aos serviços de saúde e às práticas de promoção à saúde e prevenção das doenças (Malta et al 2017).

Dessa forma, observa-se a correlação entre a maior incidência do SARS-CoV-2 e áreas de pobreza urbana e rural. A falta de políticas públicas de qualidade e do suporte de proteção coletiva dessas áreas, agrava a disseminação e aumenta a letalidade da doença nessa parcela da população. Por outro lado, as medidas de segurança potencializaram a pobreza da população, uma vez que um grande contingente da população perdeu o emprego ou meios de subsistência (Fiocruz 2020). 
Durante a pandemia, pacientes com DCNTs reduziram a prática de atividade física, aumentaram o hábito de assistir televisão e diminuíram o consumo de vegetais (Malta et al 2020).

Estudos afirmam que a inatividade física é um grave problema de saúde pública, sendo responsável por mais de 3 milhões de mortes por ano pelo mundo. Estima-se que 1/3 da população mundial com idade acima de 15 anos não realize pelo menos 150 minutos de atividade física por semana como recomendado pela OMS. Durante a pandemia, as restrições contaram com o fechamento das academias, aumentando a parcela da população sedentária (Pitanga et al 2020).

A prática regular de atividade física é uma medida de prevenção para o desenvolvimento de diversas doenças crônicas, por associar-se com o fortalecimento do sistema imunológico através do aumento da concentração de leucócitos. Dependendo da intensidade e duração da atividade, pode ocorrer uma imunossupressão durante um período de 3 a 72 horas após o exercício, devido uma brusca cessação da concentração de leucócitos em exercícios de grande intensidade e duração. Dessa forma, a American Heart Association (AHA), recomenda praticar ao menos 30 minutos de atividade física aeróbica de intensidade moderada cinco vezes por semana, totalizando 150 minutos por semana. Além de reforçar o sistema imunológico, o exercício físico é capaz de reduzir a ansiedade comum à época de pandemia através da liberação de neurotransmissores como endorfina e serotonina (Júnior 2020).

A OMS e o American College of Sports Medicine (ACSM), estimularam a estratégia de orientação à prática de atividade física de forma online, permitindo que a população pudesse fazer exercícios durante o isolamento social. Tal prática não só promove benefícios ao sistema imunológico como também beneficia os aspectos biopsicossociais. Dessa forma, podemos perceber que as plataformas digitais se tornaram um meio alternativo de promoção à saúde durante a pandemia da COVID-19. (Guimarães et al 2020).

A nutrição é crucial para o desenvolvimento e saúde humana. Está envolvida no crescimento, resposta imunológica e uma expectativa de vida maior, sendo capaz de promover saúde e prevenir doenças. Considerando que o desenvolvimento e agravo de DCNTs está relacionado com hábitos alimentares ruins, conclui-se que faz parte do tratamento a mudança do comportamento ao consumir mais alimentos com nutrientes do que alimentos processados e ultraprocessados, como têm acontecido de forma exponencial (Gois et al 2020).

Considerando que o vírus SARS-CoV-2 causa maiores injúrias à pacientes com DCNTs, torna-se necessário desenvolver ações em educação em saúde, voltadas para hábitos de vida e alimentação saudável e uso correto da medicação para controle das doenças crônicas (Estrela et al 2020).

Práticas saudáveis estão correlacionadas com maiores chances de adesão a outros comportamentos protetivos, como ocorre para os principais fatores de risco para doenças crônicas não transmissíveis. Em suma, indivíduos que se alimentam melhor, não fumam, consomem pouca ou nenhuma bebida alcoólica e praticam regularmente atividades físicas, são mais inclinados a adotar as medidas de proteção individual durante a pandemia da COVID-19 (Peixoto et al 2020).

A educação em saúde deve ser feita com informações e orientações acessíveis à população para que não ocorra as mensagens falsas (fake news) que circulam na internet e são fatores agravantes da desinformação e consequentemente, do agravo da transmissão. As mensagens devem ser claras e precisam atingir o maior número de pessoas para que, quando orientadas, possam multiplicar a informação de qualidade e propagar as medidas cabíveis para o melhor controle da doença (Sales et al 2020).

$\mathrm{O}$ isolamento social e a restrição à residência levaram à busca por entretenimento ligado à maior utilização de equipamentos visuais e dificultou a prática de atividades físicas, sendo urgente a necessidade da orientação sobre métodos alternativos de atividade física nas condições atuais, encorajando práticas mais saudáveis (Souza Filho e Tritany 2020).

O cenário atual da pandemia reforçou a necessidade de aproximar a Saúde Coletiva com a prática e ensinamentos da educação física. Durante a pandemia e o isolamento social, as práticas de atividade física foram proibidas pelas políticas do estado e posteriormente permitida por ser considerada atividade essencial. Apesar da discussão acerca do decreto, é indiscutível que a atividade física teria um efeito protetor na doença, pois sua prática melhoraria o sistema imunológico (Loch et al 2020). 
Os micronutrientes têm um papel vital no sistema imunológico. A resposta imune fica comprometida quando o estado nutricional não está adequado, predispondo os indivíduos a infecções. Portanto, durante uma pandemia onde é de interesse global manter as melhores condições de saúde para população, devemos atentar às necessidades diárias de micronutrientes, garantindo que a população receba alimentos preferencialmente minimamente processados (Gois et al 2020).

\section{Considerações finais}

Reitera-se o necessário desenvolvimento de ações educativas em saúde voltadas para hábitos de vida, alimentação saudável e uso correto da medicação para controle das doenças crônicas não transmissíveis (DCNTs).

\section{Declaração de conflito de interesse}

Os autores declaram que não há conflitos de interesse.

\section{Financiamento}

Esta pesquisa não recebeu nenhum apoio financeiro.

\section{Referências}

Azevedo ALS de, Silva RA da, Tomasi E, Quevedo L de Á (2013) Doenças crônicas e qualidade de vida na atenção primária à saúde. Caderno de Saúde Pública 29:1774-1782.

Estrela FM, Cruz MA, Gomes NP, Oliveira MAS, Santos RS, Magalhães JRF, et al. (2020) COVID-19 e doenças crônicas: impactos e desdobramentos frente à pandemia. Revista Baiana de Enfermagem 34:e36559.

Fiocruz (2020) Boletim socioepidemiológico da covid-19 nas favelas: análise de frequência, incidência, mortalidade, e letalidade por covid-19 em favelas cariocas.

Gois BP de, Pereira A, Lopes KLS, Corgosinho FC (2020) Suplementação e alimentação adequada no contexto atual da pandemia cau sada pela COVID19. Revista Interdisciplinar da Universidade Federal do Tocantins 7:89-96.

Guimarães JAC, Guerra PH, Ueno DT, Christofoletti AEM, Nakamura PM (2020) Estudo transversal sobre uso de ferramentas virtuais para orientar a atividade física durante a COVID-19. Revista Brasileira de Atividade Física \& Saúde.

Júnior LCM (2020) Alimentação saudável e exercícios físicos em meio à pandemia da COVID-19. Boletim de Conjuntura 3:9.

Loch MR, Rech CR, Costa FFF da (2020) A urgência da saúde coletiva na formação em educação física: lições com o CoVID-19. Ciência \& Saúde coletiva 25:3511-3516.

Malta DC, Bernal RTI, Lima MG, Araújo SSC de, Silva MMA da, Freitas MI de F, Barros MB de A (2017) Doenças crônicas não trans missíveis e a utilização de serviços de saúde: análise da Pesquisa Nacional de Saúde no Brasil. Revista de Saúde Pública 51 (Supl1):4s.

Malta DC, Gomes CS, Barros MB de A, Lima MG, Almeida W da S de,Nogueira de Sá ACMG, et al. (2020) Doenças crônicas não transmissíveis e mudanças nos estilos de vida durante a pandemia de covid-19 no Brasil. Revista Brasileira de Epidemiologia. doi: 10.1590/1980-549720210009

Ministério da Saúde (2020) Guia de Vigilância Epidemiológica: Emergência de Saúde Pública de Importância Nacional pela Doença pelo Coronavírus 2019.

Peixoto SV, Nascimento-Souza MA, Mambrini JV de M, Andrade FB de, Malta DC, Lima-Costa MF (2020) Comportamentos em saúde e adoção de medidas de proteção individual durante a pandemia do novo coronavírus: iniciativa ELSI-COVID-19. Cadernos de Saúde Pública 36:e00195420.

Pitanga FJG, Beck CC, Pitanga CPS (2020) Inatividade física, obesidade e COVID-19: perspectivas entre múltiplas pandemias. Revista Brasileira de Atividade Física \& Saúde. doi: 10.12820/rbafs.25e0114

Sales CMM, Silva AID, Maciel LNM (2020) Vigilância em saúde da COVID-19 no Brasil: investigação de contatos pela atenção primária em saúde como estratégia de proteção comunitária. Revista Epidemiologia e Serviços de Saúde 29:2020373.

Souza Filho BAB de, Tritany EF (2020) Covid-19: importância das novas tecnologias para a prática de atividades físicas como estratégia de saúde pública. Caderno de Saúde Pública 36:e00054420.

Teixeira MRM (2020) A pandemia de covid-19 e as doenças crônicas não transmissíveis (DCNT). Divisão de Vigilância de Doenças e Agravos Não Transmissíveis.

World Health Organization (2020) The impact of the COVID-19 pandemic on noncommunicable disease resources and services: results of a rapid assessment. Geneva: World Health Organization. 